\title{
Adapting product and supply chain systems under severe uncertainty
}

\author{
Lalit Patil and Kazuhiro Saitou, Senior Member, IEEE
}

\begin{abstract}
Currently, adaptation in a virtual enterprise is mostly reactive in response to variations that have already occurred. In order to remain profitable in the highly competitive global market, it is highly desirable to proactively adapt in anticipation of unplanned, but foreseeable high impact events, while still maintaining competitive product quality, cost, and lead-time. This paper approaches the problem by developing a unified model of product-supply chain systems based on networks of design-supplier pairs, and decision models of adaptation in the event of a jump based on real options theory. Application to a single component, single echelon supply chain is discussed.
\end{abstract}

\section{INTRODUCTION}

G LOBALIZATION is leading to new paradigms for I manufacturing enterprises. Manufacturers need to increase productivity and quality, and decrease costs and time to the global markets. As a result, the general trend is to move toward a virtual enterprise with global supply chains. This exposes a manufacturing enterprise to new uncertainties with high potential impacts, positive or negative, on its products and supply chains:

- Unplanned unforeseeable events e.g., earthquakes, hurricanes, civil wars, terrorist attacks, stock market crushes, railway accidents, and oil price raises.

- Unplanned, foreseeable events e.g., expected stricter environmental regulations on vehicle emission and plant operation in China, or building of new ports.

Enterprises usually use a reactive approach to adapt to such variations beyond their robustness. In order to remain profitable, it is desirable to adapt proactively in anticipation of unplanned, but foreseeable high impact events.

In this context, as mentioned in [1], "there is a considerable motivation for companies to implement a simultaneous consideration of supply chain design and product design, since once a product design is finalized, it is relatively costly to identify and implement modifications." In treating product design and its supply chain as a system,

Manuscript received March 20, 2003. Patil was partially funded by the University of Michigan's PLM Alliance and National Science foundation award 0653838 .

Lalit Patil (phone: +1-734-763-4056; fax: +1-734-647-3170; email: lpatil@umich.edu) and Kazuhiro Saitou (email: kazu@umich.edu) are with the Mechanical Engineering Department at the University of Michigan Ann Arbor, MI 48109 USA. which undergoes adaptations as a whole, there is a need to answer the following question:

How should a manufacturing enterprise adapt both its product design and supply chain simultaneously to remain competitive (high quality, low cost, low lead-time) in a situation when the variation in the operating environment is beyond its robustness limits?

This paper develops the overall framework and proposes a procedure to obtain decision models to address the abovementioned issue. Section II explains the concept of adaptation of the product and supply chain system. After presenting the basic approach to evaluate the flexibility for adaptation in Section III, related work is analyzed in Section IV. Section V approaches the problem by developing a unified model of product-supply chain systems based on networks of component design-supplier pairs, and decision models of adaptation in the event of jump, based on real options theory. Sections VI and VII provide details on the application to a single component single echelon product and supply chain system. Finally, the paper concludes with a discussion on possibilities for future work in Section VIII .

\section{ADAPTATION OF PRODUCT-SUPPLY CHAIN SYSTEM}

We explain the concept of adapting the product designs and supply chains using the illustration in Figure 1on page 2. Consider a fictitious smartphone manufacturer, GoBluePhone with a product-supply chain system consisting a product design, $D$ and corresponding supply chain network, $S$. Assume that $D$ consists of three components, viz., the LCD screen $\left(D_{1}\right)$, the wireless card $\left(D_{2}\right)$ and the cover $\left(D_{3}\right)$. Let $S_{1}, S_{2}$ and $S_{3}$ represent three different suppliers that supply corresponding elements of the design.

The design is robust enough to account for small uncertainties, represented by bounded intervals, in the design parameters. Suppose there is a forthcoming environmental regulation that would require less Hexavalent Chromium $\left(\mathrm{Cr}^{6+}\right)$ in the cover, $D_{3}$. The current supplier, $S_{3}$ is capable of producing $\mathrm{D}_{3}$ with amount of $\mathrm{Cr}^{6+}$ between GoBluePhone's internal requirement, $\mathrm{Cr}_{\text {goBlue }}$ and the current regulatory limit, $C r_{\text {curLimit. }}$ Assume that GoBluePhone has a way of estimating the expected value, $\mathrm{E}\left[\mathrm{Cr}_{\text {newLimit }}\right]$ of the new limit on $\mathrm{Cr}^{6+}$. Now, GoBluePhone has at least three alternatives (Figure 1), each of which comes at a certain quality loss, cost or time delay. If $C r_{\text {goBlue }}<\mathrm{E}\left[C r_{\text {newLimit }}\right]<C r_{\text {curLimit }}$, and if the quality, cost and lead time remain within acceptable ranges after the regulatory changes, then GoBluePhone's current product-supply chain system is robust (Alternative 1 in Figure 1). However, if $\mathrm{E}\left[\mathrm{Cr}_{\text {newLimit }}\right]<C r_{\text {goBlue }}$, then 


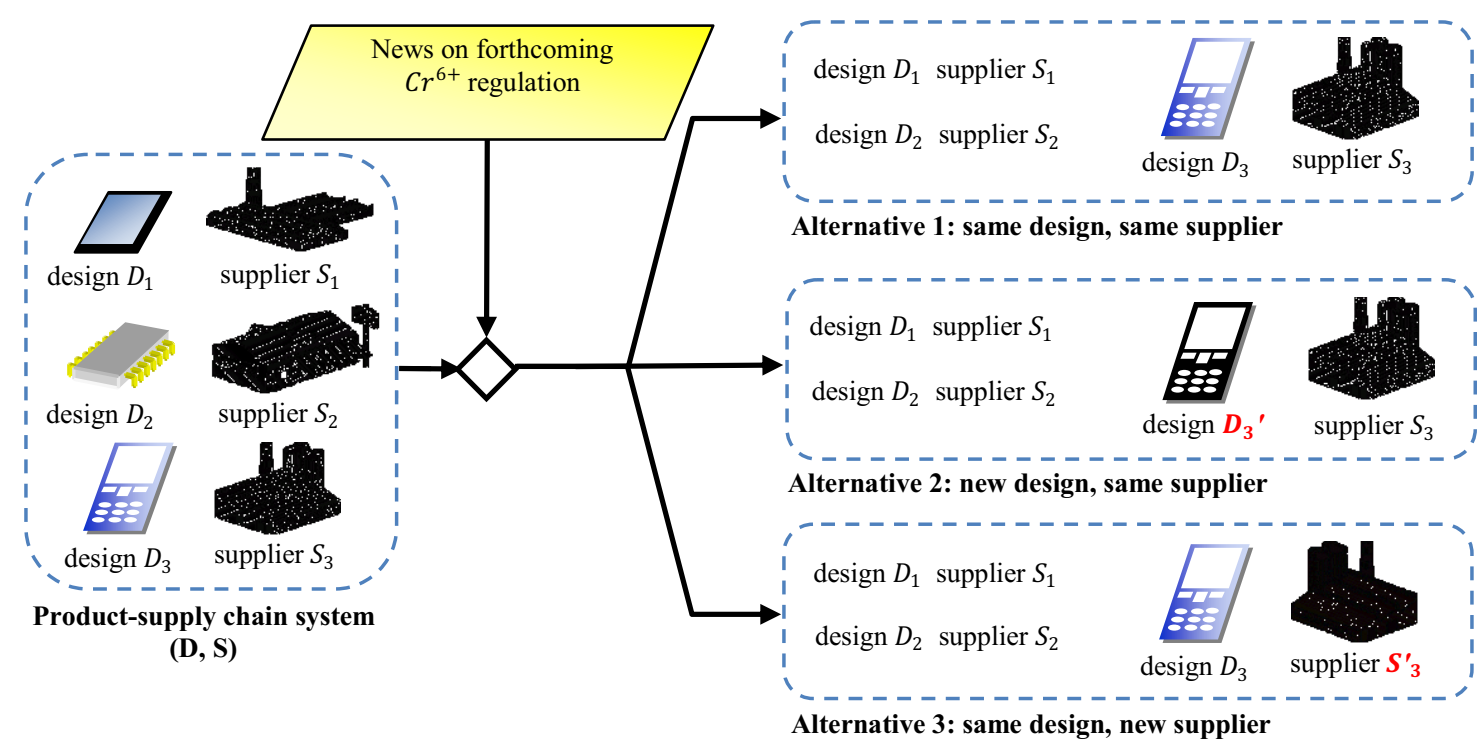

Figure 1 Under severe uncertainty, a product-supply chain system $(D, S)$ might have to adapt to available alternative. If there is no need to adapt, then the system is robust

GoBluePhone should adapt to a new product design-supply chain system. It can adopt to: a new design, $D_{3}^{\prime}$ and keep the same supplier, $S_{3}$ (Alternative 2 in Figure 1), a new supplier, $S_{3}{ }^{\prime}$, who can supply the same design, $D_{3}$ below the new regulatory limit (Alternative 3 in Figure 1), or a new design and a new supplier (not shown in Figure 1 for simplicity). This decision depends on quality loss, cost increase, time delay due to adaptation, and accuracy of $\mathrm{E}\left[\mathrm{Cr} r_{\text {newLimit }}\right]$.

This concept can be extended to a larger number of components and corresponding supply chains in the context of other high-risk factors with severe uncertainties. This GoBluePhone example highlights following questions:

1. How should an enterprise determine the best alternative for the ability to meet the quality, cost and lead time requirements, and if it is indeed worth adapting?

2. As a part of (1) above, how should the enterprise decide the time to actually implement the alternative?

\section{REAL OPTIONS APPROACH FOR ADAPTATION}

This section provides an overview of real options theory as compared to the Net Present Value (NPV) rule, a conventional model for enterprise-wide decision-making.

The NPV rule states that one should calculate the expected value of the future returns and compare it with the present value of the alternative to make a decision. If $\mathrm{V}(P(t))$ is value, at time, $\mathrm{t}$ of the performance of a productsupply chain system, then $\mathrm{NPV}=\mathrm{E}[\mathrm{V}(P(t))]-\mathrm{V}(\mathrm{P}(0))$ where, $\mathrm{E}[\mathrm{V}(P(t))]$ is expected value of the performance of the product-supply chain system at time $t$. The NPV rule has been applied to decision making in financial investments, product design [1], and project management [2].

The NPV rule assumes that either the investment decision is completely reversible without any loss, or, even if the decision is irreversible, it is a "now or never" choice.
Consequently, it suggests early investment to implement the alternative fully. However, it is possible that between now and time, $t$ and all the investment will be lost, because expected values could be incorrect. Instead of making the final decision this early, the enterprise could wait (additional cost, e.g., loss to a competitor, time delay of project, etc.) for additional information that could reduce the uncertainty.

The real options theory overcomes this limitation by applying financial options theory to quantify the value of flexibility [3]. A real option is the ability to implement an alternative, with no obligation to implement it. The cost of such an option is significantly less than that of actual implementation. Consequently, the real options theory suggests delaying investment to implement an alternative fully until uncertainty is eliminated or reduced significantly. After an initial investment, the management can wait for more news, and then decide if it should fully implement the alternative. We apply the real options approach to value the flexibility of alternative designs and suppliers for adaptation.

Calculating option values of an alternative independent of other alternatives, enables determining the better one and, possibly, the right time to invest in it. However, this approach cannot be used to decide if we can/should switch among multiple alternatives at various time states. In addition, decision-making in the real world is path dependent, i.e., the decision on an alternative at a future date may depend on the path taken by another alternative until then. Therefore, this paper considers all the alternative product-supply chain systems as part of one "enterpriselevel" system, and then applies real options in this system.

\section{RELATED WORK}

In [4], the authors focus on designing a six-sigma supply chain that aims for robustness of achieving the delivery time. They do not consider costs or other criteria. In [5], the authors discuss choosing suppliers to minimize shortfall of products in various disruption scenarios. The overall idea is to match demand and supply using only costs as the criteria. 
In [2], technological engineering design is linked with other business decisions of the firm to enable resource allocation by considering the resources to invest in different products. Several alternatives are compared to obtain a "most robust" design. However, there is no consideration for creating a new product mix if the existing one is not "good enough" in an unplanned event.

Models to design supply chains that perform well when the variations are beyond the limits of robustness are presented in [6]. It states that supply chain optimization models have traditionally assumed complete certainty of information, and argues the need for models that incorporate various forms of uncertainty into strategic decisions about supply chain design.

A model to help firms value dual-sourcing strategies, especially in the context of supply-chain disruptions, is presented in [7]. It uses supplier costs as the design criteria using geometric Brownian motion with Poisson jumps to model the underlying asset.

In [8], the authors study the option value of being able to switch between producing or outsourcing an item in the context of price and cost uncertainties. They further study and present approaches to capture the difference between instantaneous and the time-delayed implementations [9].

The challenges of identifying and using real options in engineering systems against the financial options and real options on engineering systems are discussed in detail in $[10,11]$. While explaining that real options in systems are related to design flexibility, they present a systematic approach to model them and approach the solutions. In our research, we shall derive from this systematic framework.

Our research differentiates itself from existing efforts by the following:

- We consider the need to adapt the product-supply chain system, i.e., both its design and its supply chain simultaneously, to remain profitable in a situation when the variation of the performance-affecting parameters is beyond the limits of robustness.

- We simultaneously consider multiple criteria: quality (design) and cost and time (supply chain) in adaptation.

\section{OVERALL PRODUCT-SUPPLY CHAIN SYSTEM MODEL}

We propose a product-supply chain system to be represented as a pair $(D, S)$, where $D$ is the design of the product consisting of $n$ components. $S$ represents the supply chain consisting of suppliers for each of the components. In other words, a product-supply chain system is made of $n$ pairs of component design and supplier, represented by $\left(D_{i}\right.$, $\left.S_{i}\right) \in D \times S, 1 \leq i \leq n$, which we shall refer to as componentsupplier subsystem. The following sections discuss the representation of the overall performance of the productsupply chain system and severe uncertainties.

\section{A. Performance of a product-supply chain system:}

Assume that $R(t)$ represents the ability to meet the amount of substance regulation (say Hexavalent Chromium in a component), during a time interval beginning at time, $t$. The performance of a component-supplier subsystem, $\left(D_{i}, S_{i}\right)$ during a time interval beginning at time, $t$ is given by

$$
P_{i}\left(t, R_{i}(t)\right)=f\left(Q_{i}\left(t, R_{i}(t)\right), C_{i}\left(t, R_{i}(t)\right), T_{i}\left(t, R_{i}(t)\right)\right)(1)
$$

where $Q_{i}\left(t, R_{i}(t)\right), \quad C_{i}\left(t, R_{i}(t)\right), \quad$ and $\quad T_{i}\left(t, R_{i}(t)\right)$ are, respectively, the abilities to meet the quality, cost, and lead time requirements. The overall performance of the productsupply chain system is some aggregation of performances of component-supplier subsystems:

$$
P\left(t, R_{i}(t)\right)=g\left(P_{1}\left(t, R_{i}(t)\right), P_{2}\left(t, R_{i}(t)\right), \ldots, P_{n}\left(t, R_{i}(t)\right)\right)
$$

\section{B. Severe uncertainties as jump processes:}

We propose to represent severe uncertainties (infrequent, but discrete "events") by using jump processes $[12,13]$. For example, a Poisson process is subject to jumps of fixed or random size, for which the arrival times follow a Poisson distribution. Therefore, some form of jump-diffusion process captures the combination of regular uncertainties and jumps. Assume that the ability to meet the regulations, $R(t)$ in response to the sudden change in the regulation follows a Poisson jump process: $d R(t)=d q$, where $d q$ is the increment of the Poisson process with mean arrival rate, $\lambda$ and some predicted amplitude, $\varphi$. If $R$ is non-positive, i.e., the product is unable to meet the regulation now, then, the enterprise would have to adapt so that the overall performance, $P(t)$ is within desired limits.

The next section presents the development of appropriate decision models in a single-echelon supply chain with single component products.

\section{APPLICATION TO SINGLE-ECHELON SUPPLY CHAINS WITH SINGLE COMPONENT PRODUCTS}

For a single component, single echelon supply chain, the manufacturer looks for an alternative single componentsupplier combination. Let $P_{M}$ be the ability to meet manufacturer's (pre-defined) performance requirement.

\section{A. Model the firm's overall expected performance}

Assume that the manufacturer can predict the required ability to meet expected performance over a finite time horizon $(0, \mathrm{~T}]$. It can achieve this by predicting the demand, supply and its plan for the high-impact change to remain profitable. Assume that this is a mean-reverting process with Poisson shock transmitted from jump in regulations:

$$
d P_{M}=\eta_{M}\left(\overline{P_{M}}-P_{M}\right) d t+\sigma_{M} d z_{M}-d q
$$

The component, $\eta_{M}\left(\overline{P_{M}}-P_{M}\right) d t+\sigma_{M} d z_{M}$ of equation (3) represents the mean-reverting process, the normal expected behavior. Here, $\eta_{M}$ represents the rate of return to the mean, $\overline{P_{M}}$, and $\sigma_{M}$ represents the deviation. These parameters can be obtained from archival data or specific requirements. $d z_{M}$ is standard Brownian motion.

The last term, $d q$ in equation (3), captures jump due to the jump in regulations. If a sudden changing event occurs, $q$ drops by a fixed percentage $\varphi: 0 \leq \varphi \leq 1$ with probability 1 .

Thus, equation (3) says that $P_{M}$ will fluctuate as a mean reverting motion with mean, $\overline{P_{M}}$ and some variance, $\sigma_{M}$, but over each time interval $d t$, there is a small probability 
$\lambda d t$ that it will drop to $(1-\varphi)$ times its original value, after which it continues fluctuating until another event occurs.

We construct a discretized events tree using a trinomial expansion to capture this process. Assume that the current state is at time, $t$, and the future state is at the time, $t+\Delta t$ after a time interval, $\Delta t$. For the mean-reversion part of the process represented by equation (3), the expected value of $\mathrm{P}_{M, t+\Delta t}$ given $\mathrm{P}_{M, t}$, is given by

$$
\mathrm{E}\left[\mathrm{P}_{M, t+\Delta t}\right]=\mathrm{P}_{M, t} e^{-\eta_{M} \Delta t}+\overline{P_{M, t}}\left(1-e^{-\eta_{M} \Delta t}\right)
$$

The variance is given by

$$
\operatorname{Var}\left(\mathrm{P}_{M}\right)=\frac{\left(1-\mathrm{e}^{2 \eta_{M} \Delta t}\right) \sigma_{M}{ }^{2}}{2 \eta_{M}}
$$

In discrete terms, in the absence of a jump, the performance moves up by a factor of $u$ to $P_{M, t+\Delta t}^{+}=u . P_{M, t}$, or down by a factor of $d$ to $P_{M, t+\Delta t}^{-}=d . P_{M, t}$.

Assume the probability of moving up at any given time instant, $t$ is given by $q_{M, t}{ }^{+}=0.5$, and the probability of a drop in the performance is given by $q_{M, t}^{-}=1-q_{M, t}^{+}=0.5$.

Thus,

$$
q_{M, t}^{+} u \cdot P_{M, t}+q_{M, t}^{-} d \cdot P_{M, t}=E\left[P_{M, t+\Delta t}\right]
$$

and

$$
u^{2} q_{M, t}^{+}+d^{2} q_{M, t}^{-}-\left(u q_{M, t}^{+}+d q_{M, t}^{-}\right)^{2}=\operatorname{Var}\left(\mathrm{P}_{M}\right)
$$

From the above set of equations, we get

$$
\begin{aligned}
& u=0.5 \times \frac{E\left[P_{M, t+d t}\right]}{P_{M, t}}+\sqrt{\operatorname{Var}\left(P_{M}\right)} \\
& d=0.5 \times \frac{E\left[P_{M, t+d t}\right]}{P_{M, t}}-\sqrt{\operatorname{Var}\left(P_{M}\right)}
\end{aligned}
$$

Now, consider the jump process that captures the highimpact events. The probability of the occurrence of the jump in the time interval $\Delta t$ is given by $\lambda \Delta t$. Therefore, the probability of the occurrence of the normal behavior captured by the mean-reversion process is given by $1-\lambda \Delta t$. Accordingly, the 3 different future states of the performance at time $t+\Delta t$ are as shown in Figure 2 .

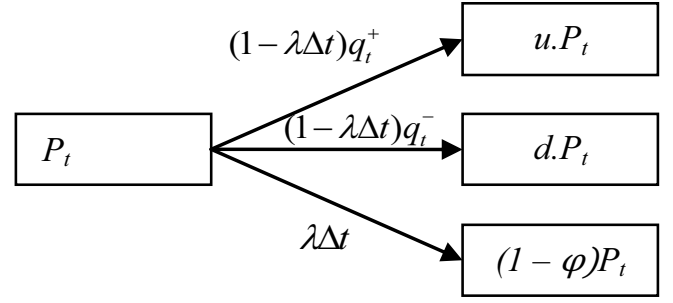

Figure 2 Three events are possible from current state at time $t$ to next state at time $t+\Delta t$

After using the above procedure to model the manufacturer's overall expected performance and its variation along the time horizon, we identify available alternatives that can be executed by the manufacturer.

\section{B. Executable alternatives}

We can identify the set of alternative component-supplier subsystems and the costs to adapt. These are one or more of the following three types of control variables at any time, $t$ :
- Abandon project, i.e., control variable $\left(v_{t, 0}\right)=1$. This can be done at the return of some value $A$.

- Use current system, $\left(\boldsymbol{D}_{1}, \boldsymbol{S}_{1}\right)$ i.e., control variable $\left(v_{t, 1}\right)$ $=1$. This is at no additional relationship cost to the firm.

- Use one alternative component-supplier subsystem, $\left(\boldsymbol{D}_{\boldsymbol{i}}, \boldsymbol{S}_{\boldsymbol{i}}\right)$, i.e., control variable $\left(v_{t, i}\right)=1$ This is the option to adapt to one of the alternatives. It comes at an additional cost, $K$, which we assume includes the cost to break off the current relationship.

The set of the control variables at any time, $t$ is denoted by $v_{t}$. Now, we model the performance of every componentsupplier subsystem, including the current.

\section{Model the component-supplier performance}

For any component-supplier subsystem, $\left(D_{i}, S_{i}\right)$, we assume that each of $Q_{i}, T_{i}, C_{i}$, the ability to meet the quality, time and cost requirement respectively, follows a meanreverting jump diffusion process with Poisson shock transmitted from the jump in regulations. For simplicity, we neglect the compound nature of the Poisson process. Thus,

$$
d X^{w}=\eta^{w}\left(\overline{X^{w}}-X^{w}\right) X^{w} d t+\sigma^{w} X^{w} d z^{w}-X^{w} d q
$$

where $w \in\{1,2,3\} ; X^{1}=Q, X^{2}=C, X^{3}=T$ Then, $\eta^{w}$ represents

the rate of return to the mean ability, $\overline{X^{w}}$ to meet the requirement and $\sigma^{w}$ represents the deviation. These parameters can be obtained from archival. Then, $d z^{w}$ are standard Brownian motion satisfying the condition:

$$
\text { Covariance }\left[d z^{w}, d z^{v}\right]=\rho^{v w} d t(v \neq w)
$$

$d q$ and $d z^{w}$ are independent of each other.

While each component-supplier subsystem may have its own function to capture the relationships between the abilities to meet the quality, cost, and time requirements, assume that for any component-supplier subsystem $\left(D_{i}, S_{i}\right)$ :

$$
P_{i}(t)=\operatorname{average}\left(Q_{i}(t), C_{i}(t), T_{i}(t)\right)
$$

In order to create a combined product-supply chain model, we create discrete events trees that capture the variation along the ability to meet each of the quality, cost and time requirements as discussed in Section VI.A. In order to capture the states that match exactly with those in the tree of the expected ability to meet the performance, we use the same probability values of $q_{t}^{+}, q_{t}^{+}$for the mean-reversion part of each process. Since the arrival rate of the jump is the same across all parameters, we use the same values of time intervals, $\Delta t$ to obtain the same values for the probabilities of the three future states given the state at any time, $t$.

\section{Real options analysis}

Let $V\left(P_{M}\right)$ be the value of the ability to meet the performance, $P_{M}$ that the manufacturer expects from its product design-supply chain system. The value of the ability to meet the performance for any component-supplier subsystem $\left(\mathrm{D}_{i}, \mathrm{~S}_{i}\right)$ is $V\left(P_{i}\right)$. The manufacturer's goal is to maximize the system's ability to meet its expected ability. The basic idea is to compare the values of the performances of the alternative component-supplier subsystems to the value of the expected performance from the manufacturer, 
and then choose the one with the highest payoff. This must include a flexible decision-making process along time, i.e., the decision to implement or continue holding the option is made at various time instants, $t$ 's along the time horizon.

In the real options framework, we work on the decision tree that captures the variation of $P_{M}$ (See, Section VI.A). The standard dynamic programming approach that is commonly applied to real options analysis is used. We start at the end of the tree and analyze the optimal execution of the abandonment, continuation, or switch option at each of the final nodes. Let $x_{t}$ denote the state at time $t$. The aim is to choose the optimal set of control variables, $v_{t}$ (See, Section VI.B) at each time instant, $t$ of interest, such that the value of the expected payoffs at the initial time is maximized.

Thus, let $F_{t}\left(x_{t}, v_{t}\right)$ represent the total value of all the future payoffs if the manufacturer follows the optimal decision process from the state. Let $\pi\left(x_{t}, v_{t}\right)$ represent the difference between the product design-supply chain system's ability to meet the performance and the manufacturer's expected ability. Therefore, if $\gamma$ is the discount rate, the firm has to choose $v_{t}$, such that

$$
F_{t}\left(x_{t}, v_{t}\right)=\max _{v_{t}}\left\{\pi\left(x_{t}, v_{t}\right)+\frac{E_{t}\left[F_{t+1}\left(x_{t+1}, v_{t+1}\right)\right]}{1+\gamma}\right\}
$$

The payoff for a node $j$ at the end of the last time step, $N$, i.e., at the expiration of the decision-making time, is given by

$$
F_{j, N}=\max \left\{\mathrm{A}, V_{j, N}\left(P_{1}\right), V_{j, N}\left(P_{2}\right)-K, \ldots\right\}-V_{j, N}\left(P_{M}\right)
$$

In equation (13), if the payoff is $A-V_{j, N}\left(P_{M}\right)$, the project should be abandoned, i.e., $v_{t, 0}=1$ due to the lack of a viable alternative. If the payoff is $V_{j, N}\left(P_{1}\right)-V_{j, N}\left(P_{M}\right)$, the current supplier should be continued, i.e., $v_{t, 1}=1$. If the payoff is $V_{j, N}\left(P_{2}\right)-K-V_{j, N}\left(P_{M}\right)$, the firm should adapt to the second supplier, and so on. Depending on the alternative that leads to the best payoff, values of the control variables at the last time step are assigned.

Then, we work back to the first node of the tree (the current valuation time) along the branches of the decision tree (Section VI.A). Nodes at which execution of one of the three options is optimal are identified. The payoff at any time step, $n-1<N$ is given by

$$
F_{i, n-1}=\max \left\{\mathrm{A}, V_{i, n-1}\left(P_{1}\right), V_{i, n-1}\left(P_{2}\right)-K, P V_{n}\right\}-V_{j, n-1}\left(P_{M}\right)
$$

$P V_{n}$ represents the payoff if the manufacturer decides to not exercise any option, but to continue exploring/holding it at the time $n-1$. This is discounted weighted sum of the future payoffs using the probabilities at the weights.

Thus, at every stage, the manufacturer can decide to either adapt completely, or completely continue with the current alternative, or continue to hold to the option (i.e., continue with the current supplier and keep exploring the relationship with the alternatives), or completely abandon the project.

\section{COMPUTATIONAL RESUltS}

This section presents results from application of the procedure to a single component, single echelon productsupply chain system.

The manufacturer expects the system's ability to meet the performance to vary according to the mean-reversion with jump model given by equation (3) with the following parameters:

$$
P_{0}=100 ; \overline{P_{M}}=100 ; \sigma_{M}=5 ; \lambda=0.01 ; \varphi=0.3 ; \eta_{M}=5
$$

Table 1 shows the parameters for the processes for the component-supplier subsystems. The intensity of the Poisson shock in regulations is same for every process. For the current design-supply chain system, $\left(D_{l}, S_{l}\right)$, the mean quality exceeds expectations; however, it drops significantly in the case of a jump in the regulation. This is because the supplier, $S_{I}$ is in a country where the shock has a direct impact. For system, $\left(D_{1}, S_{3}\right)$, the supplier, $S_{3}$ provides the current design, the quality and time that would satisfy the conditions during a jump, but the mean of the value of the ability to meet cost is very low (30). For system, $\left(D_{2}, S_{2}\right)$, the cost and time abilities are poor, but none is dramatically low. In addition, it is robust in the case of the jump in the regulations.

Table 1 Parameters for the processes for each component-supplier subsystem

\begin{tabular}{|l|l|l|l|l|l|l|}
\hline Alternative & \multicolumn{1}{|c|}{$\begin{array}{c}\text { Cost Ability } \\
(C)\end{array}$} & \multicolumn{2}{|c|}{$\begin{array}{r}\text { Time Ability } \\
(T)\end{array}$} & \multicolumn{2}{|c|}{$\begin{array}{r}\text { Quality Ability } \\
(Q)\end{array}$} \\
\cline { 2 - 7 } & $\bar{C}$ & $\varphi$ & $\bar{T}$ & $\varphi$ & $\bar{Q}$ & $\varphi$ \\
\hline$D_{1}, S_{1}$ & 100 & 0.5 & 100 & 0.3 & 110 & 0.7 \\
\hline$D_{1}, S_{3}$ & 30 & 0.2 & 110 & 0.1 & 110 & 0.1 \\
\hline$D_{2}, S_{2}$ & 60 & 0.05 & 70 & 0.1 & 110 & 0.1 \\
\hline
\end{tabular}

Furthermore:

- Variance for every process is given by $\sigma=5$

- Rate of return for each mean-reverting process, $\eta=5$

- $\quad$ Salvage value after abandoning the project $A=20$

- Cost of switching, $K=20$

- Time within which decision is made, $T=6$ months.

- Situation is evaluated after every $\Delta t=2$ months

Figure 3 shows the final optimal decision tree from the computation based on the procedure outlined in Section VI. Labels on the edges indicate a decision that the manufacturer should undertake at that node. Section VI.A explains calculations of the probabilities of moving from the current state to one of the next states in time. The tree indicates that the manufacturer should switch to alternative $\left(D_{1}, S_{3}\right)$ only at the worst possible scenario ( 3 consecutive jumps). This is a very unlikely situation with a probability of 0.000001 .

In this particular case, it is mathematically possible to suggest to not adapt even after the jump. This is because we have considered a very small 3-period example for the sake of better explanation. In addition, the high variance in comparison to the lower value of expected mean affects the decision model. Decision trees over more time steps and better data demonstrate realistic results, but are more difficult to read and present. 


\section{CONCLUSION}

Understanding how high-impact uncertain events would affect the enterprise is of paramount importance. Research efforts have traditionally focused on using single criteria, such as costs (and more recently, lead-time) as the criteria for evaluating the supply chain. In this paper, we focus on the simultaneous synthesis of product design and supplychain to handle adaptation when variation in the operating environment is beyond the robustness limits. We focus on product quality (design criteria) along with cost and lead time (supplier attributes) as drivers of the performance, by modeling them as mean-reverting jump-diffusion processes. We have demonstrated the basic framework and approach by application to a simple situation of a single component, single echelon supply chain.

Our ongoing research focuses on extensions to multi-tier supply chains and applications multi-component systems. In addition, we are working on modeling the system to incorporate the time delay between the decision to adapt and the actual adaptation.

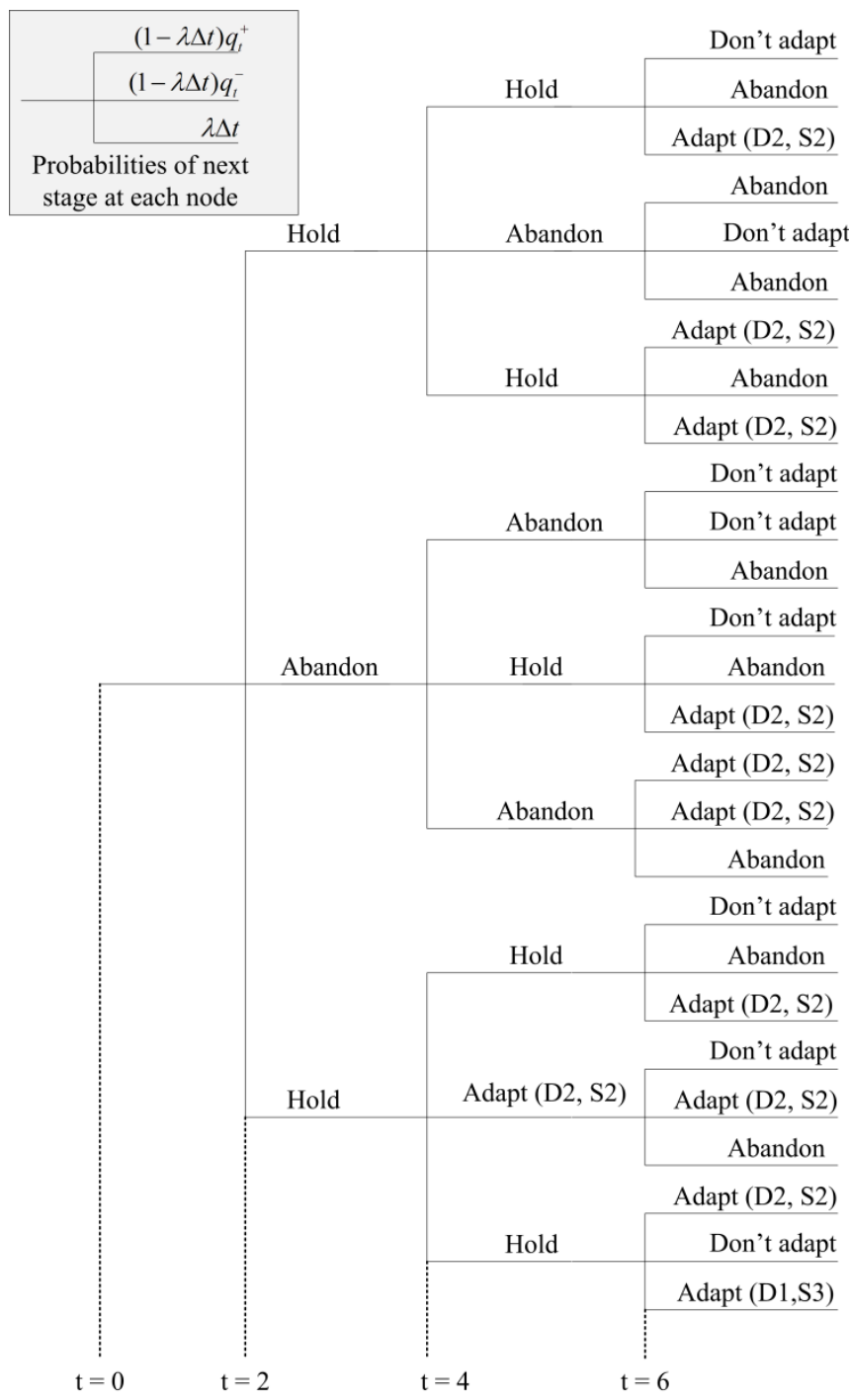

Figure 3 Resultant decision-model to proactively adapt for the example case study.

\section{REFERENCES}

[1] J. Blackhurst, T. Wu, and P. O'grady, "PCDM: a decision support modeling methodology for supply chain, product and process design decisions," Journal of Operations Management, vol. 23, pp. 325-343, April 2005.

[2] P. Georgiopoulos, M. Jonsson, and P. Y. Papalambros, "Linking optimal design decisions to the theory of the firm: The case of resource allocation," Journal of Mechanical Design, vol. 127, pp. 358-366, May 2005.

[3] A. K. Dixit and R. S. Pindyck, Investment under uncertainty. Princeton, N.J.: Princeton University Press, 1994.

[4] D. Garg, Y. Narahari, and N. Viswanadham, "Design of six sigma supply chains," IEEE Transactions on Automation Science and Engineering, vol. 1, pp. 38-57, Jul 2004.

[5] R. S. Gaonkar and N. Viswanadham, "Analytical Framework for the Management of Risk in Supply Chains," IEEE Transactions on Automation Science and Engineering, vol. 4, pp. 265-273, 2007.

[6] L. V. Snyder, "Supply Chain Robustness and Reliability: Models and Algorithms," in Department of Industrial Engineering and Management Sciences. vol. Ph.D. dissertation Evanston: Northwestern University, 2003.

S. Pochard, "Managing Risks of Supply-Chain Disruptions: Dual Sourcing as a Real Option," in Engineering Systems Division. vol. Masters Thesis Cambridge: Massachusetts Institute of Technology, 2003.

[8] H. B. Nembhard, L. Shi, and M. Aktan, "Supply chain modeling: a real options design for product outsourcing," in Proceedings of the 33rd conference on Winter simulation Arlington, Virginia: IEEE Computer Society, 2001, pp. 548552.

[9] H. B. Nembhard, L. Y. Shi, and M. Aktan, "A realoptions-based analysis for supply chain decisions," IIE Transactions, vol. 37, pp. 945-956, Oct 2005.

R. de Neufville and T. Wang, "Building Real Options into Physical Systems with Stochastic Mixed-Integer Programming," in Proceedings of the 8th Annual International Conference on Real Options Montreal, Canada, 2004.

[11] T. Wang and R. de Neufville, "Identification of Real Options "in" Projects," in 16th Annual International Symposium of the International Council on Systems Engineering (INCOSE), Orlando, FL, 2006.

[12] D. Applebaum, Lévy processes and stochastic calculus. New York: Cambridge University Press, 2004.

R. Cont and P. Tankov, Financial Modelling with Jump Processes: Chapman and Hall/CRC, 2007. 